\title{
Dynamic analysis and characterization of a nonlinear bi-stable piezo-magneto-elastic energy harvester
}

\author{
João Victor Ligier Lopes Peterson ${ }^{1, *}$, Vinicius Gonçalves Lopes ${ }^{1, * *}$, and Americo Cunha $\mathrm{Jr}^{1, * * *}$ \\ ${ }^{1}$ Universidade do Estado do Rio de Janeiro
}

\begin{abstract}
Energy harvesting is a very promising technology to provide low levels of power for small autonomous systems, which the applicability encompass a very wide range of areas, that spans from micro/nano sensors in engineering to state of art implants in medicine. The present work deals with the analysis and detailed characterization of a nonlinear bi-stable piezo-magneto-elastic energy harvester driven by a periodic external excitation. The dynamical system is studied in depth through bifurcation diagrams and basins of attraction. The level of chaoticity of the dynamical system is accessed very efficiently via the 0-1 test for chaos, which allows mapping the presence of dense regions of chaos without the help of the Lyapunov exponents.
\end{abstract}

\section{Introduction}

Energy harvesting technologies are very promising alternatives for electrical supplying of low power systems, such as medical implants, micro electro-mechanical systems (MEMS), nano electromechanical systems (NEMS), etc $[1,2]$. Such devices converts environmental energy, available for free, into electrical power to be used in the application of interest. Due to the immense possibility of applications, together with the theoretical challenges associated with the analysis of the underlying nonlinear behavior, these dynamical systems have been object of study of several recent works [3-8].

The present paper aims to investigate the nonlinear dynamics of the bi-stable piezo-magneto-elastic energy harvester by Erturk et al. [9]. In particular, it is of interest to map out whether the system response is chaotic or regular, since the nature of the response of the system has great influence on the level of energy possibly recoverable.

The dynamic system of interest is investigated via bifurcation diagrams and basins of attraction, where the latter are computed not in the traditional way, but with the aid of a binary test for chaos detection.

The rest of this paper is organized as follows. The second section introduces the harvester device of interest and the mathematical model used to analyze its dynamic behavior. Bifurcation diagrams are presented in the third section, while an analysis with $0-1$ test for chaos and basins of attraction is conducted in the fourth section. Finally, the fifth section, summarizes the paper main conclusions and contributions.

\footnotetext{
*e-mail: joao.peterson@uerj.br

**e-mail: vinicius.g.lopes@uerj.br

***e-mail: americo@ime.uerj.br
}

\section{Mathematical modeling}

\subsection{Physical model}

A schematic representation of the bi-stable piezomagneto-elastic energy harvester studied here is depicted on Figure 1. A slim ferromagnetic cantilever beam, exposed to magnetic fields effects, excites the piezo-electric material plates placed on its upper edge. The rigid structure supporting the whole ensemble is subjected to a periodic movement which drives the nonlinear system.

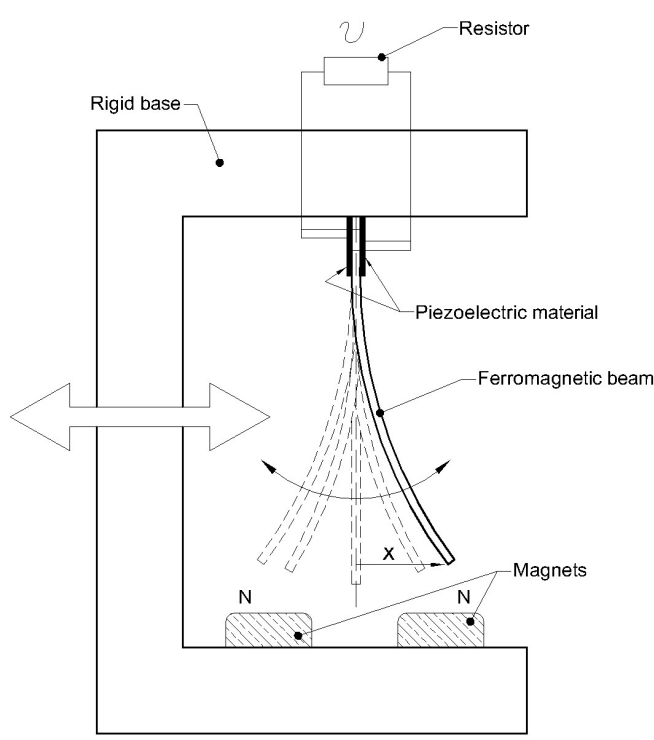

Figure 1. Schematic representation of the bi-stable piezomagneto-elastic energy harvester by [9] . 


\subsection{Model equations}

The dynamic behavior of physical system in Figure 1 evolves according to an evolution law dictated by the pair of equations

$$
\begin{gathered}
\ddot{x}+2 \xi \dot{x}-0.5 x\left(1-x^{2}\right)-\chi v=f \cos (\Omega t), \\
\dot{v}+\lambda v+\kappa \dot{x}=0,
\end{gathered}
$$

where $\chi$ and $\kappa$ represents the specific piezoelectric coupling terms, $\xi$ the mechanical damping ratio, $\lambda$ a time constant reciprocal and, finally, $f$ and $\Omega$ represent the excitation amplitude and frequency, respectively. The beam displacement $x$, its velocity $\dot{x}$ and output voltage $v$ are obtained through the integration of the initial problem value (IPV) defined by Eqs. (1) and (2) and appropriate initial conditions, using the values $\xi=0.01, \chi=0.05, \lambda=0.05$ and $\kappa=0.5$. All the aforementioned quantities are dimensionless.

\section{Bifurcation diagrams}

In order to identify if the dynamic behavior of the bistable harvester device is regular or chaotic, this section makes an analysis based on bifurcation diagrams, where the output voltage is considered as an observable of the dynamical system, which is calculated as a function of a certain control parameter (excitation force amplitude $f$ or excitation force frequency $\Omega$ ).

These diagrams are constructed increasing (forward analysis) or decreasing (backward analysis) the control parameter in a quasi-static way, plotting on the vertical axis the number of periods associated with the current solution. This analysis establishes an interval of interest for the control parameter $(f$ or $\Omega$ ), which is discretized by an uniform mesh with 1200 points. For all cases under analysis, initial displacement, velocity and voltage are set as $\left(x_{0}, \dot{x}_{0}, v_{0}\right)=$ $(1,0,0)$. Only the last $10 \%$ of the time series are considered in the construction of the diagrams.

\subsection{Fixed amplitude, variable frequency}

The first analysis considers $\Omega$ as the control parameter, observing the system dynamics in the interval $0.1 \leq \Omega \leq 1.4$, for a fixed set of forcing amplitude $f \in\left\{f_{n}=0.019+(n-1) 0.032, n=1, \cdots, 9\right\}$, as can be seen in Figure 2.

The backward diagram reveals a chaotic dynamic behavior for higher frequencies in the addressed interval and $f \geq 0.051$. Multiple period responses comes up close to the chaotic regions for $f \geq 0.179$. Regions of regular (non-chaotic) dynamics can be seen between chaotic areas.

For both diagrams, forward and backward, a spread region with discontinuities emerges on low frequencies interval, for amplitudes between $f=0.083$ and 0.211. Regular dynamics can be observed for an

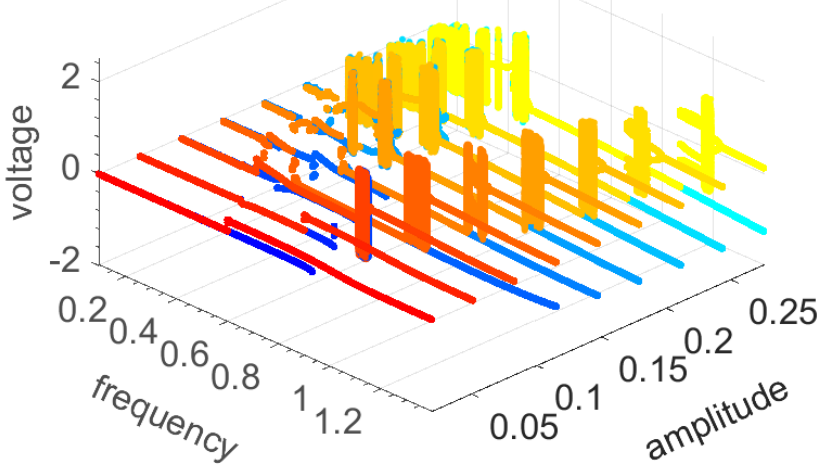

Figure 2. Bifurcation diagrams of the output voltage as function of the excitation frequency, for several values of excitation force amplitude. Forward analysis are presented blue tones, while backward analysis appear in red tones.

entire interval of frequencies only when $f=0.019$, despite of non-zero values been registered for $\Omega \geq 0.8$ on the forward diagram.

A general analysis suggests that negative regular voltages appears, in the forward diagram, spread on high frequencies interval, and at the central region on both diagrams, specially for $f \geq 0.179$. Positive response predominates for all $f$ interval on higher frequencies values over $\Omega=0.8$, with some of then also presenting chaotic patterns.

\subsection{Fixed frequency, variable amplitude}

In the second analysis $f$ is taken as control parameter, observing the system dynamics in the interval $0.05 \leq$ $f \leq 0.3$, for a set of fixed forcing frequencies $\Omega \in\left\{\Omega_{n}=\right.$ $0.1+(n-1) 0.1, n=1, \cdots, 9\}$, as can be seen in Figure 3 .

It is possible to see that regular behavior dominates the lower portion of $f$ interval, for frequencies smaller than $\Omega=0.8$, in both diagrams (forward and backward). Although, the same analysis reveals that voltage reaches zero on both cases, for frequencies $\Omega<0.5$. Regular negative values, indicating poles inversion on piezoelectric terminals, can be noted on backward diagrams for $\Omega \geq 0.6$, for almost all amplitudes analyzed.

Chaotic dynamics have a strong presence for low frequencies until $\Omega=0.5$ on both diagrams, even for amplitude from $f \geq 0.119$. Forward diagrams shows chaotic patterns emerging on the middle portion of $f$ interval for frequencies as $\Omega=0.8$ and $\Omega=0.9$, while backward diagrams remains regular, presenting a similar profile since $\Omega=0.6$. 


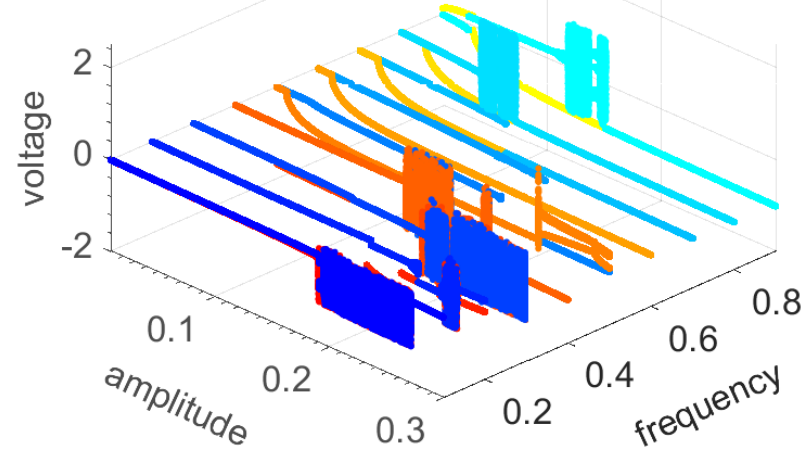

Figure 3. Bifurcation diagrams of the output voltage as function of the excitation force amplitude, for several values of excitation frequency. Forward analysis are presented blue tones, while backward analysis appear in red tones.

Other regular results are obtained for $\Omega=0.7$, where backward diagrams reveals negative voltages for $f \geq 0.03$, and in the forward diagrams for $f=\geq$ 0.11 .

The two diagrams reveal the coexistence of stable solutions for the dynamic system of interest, depending on the configuration of parameter adopted for the external excitation.

\section{Basins of attraction}

Another tool used here to characterize the system dynamic behavior of the bi-stable harvester is the $0-1$ test for chaos by Gottwald and Melbourne [10]. This statistical test uses a binary classifier $K \in\{0,1\}$ to identify the regime of the dynamics $-K=0$ means regular dynamics, while $K=1$ stands for chaotic behavior. The classifier is constructed with basis on an extension of the dynamical system to a twodimensional Euclidean group, that (asymptotically) behaves likes a Brownian motion, in case of chaotic dynamics, and generates limited trajectories for deterministic dynamics. A time series of the dynamical system is projected into an extended space and undergoes a sequence of transformations, to generate the classifier, that depends on a parameter randomly $c$. The test is repeated hundreds of times, each time with a new value of $c$, and the final classifier is given by the median of the several samples of $K$ previously generated. Further details about this test can be seen in $[10,11]$.

Figure 4 shows the projection of a basins of attraction in the displacement vs velocity plane, obtained from the 0-1 classifier, for excitation amplitude and excitation frequency values equal to $f=0.083$ and
$\Omega=0.8$, respectively. It is seen that chaos and regularity have well-defined regions, with blue representing chaotic responses and red regular ones.

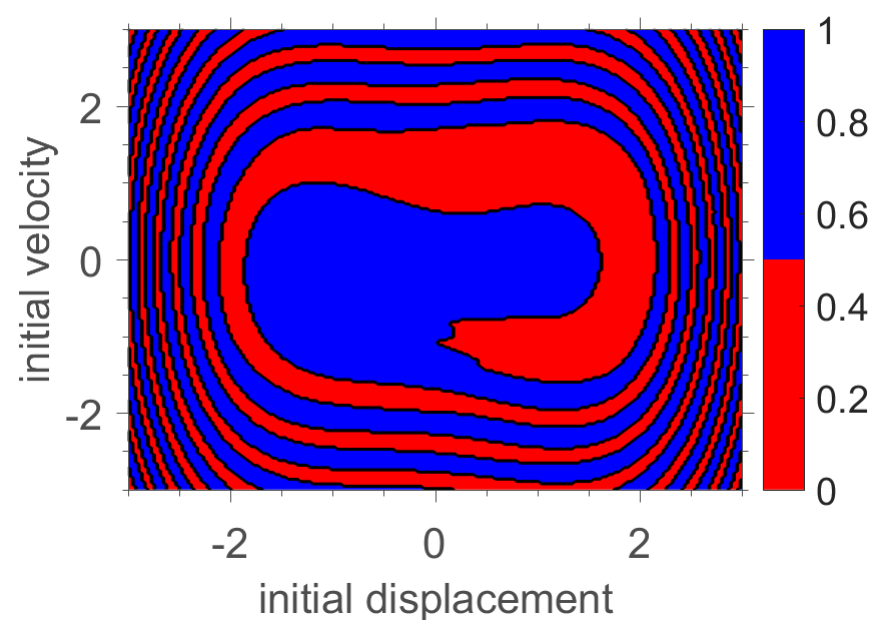

Figure 4. Projection of the basins of attraction in the displacement vs velocity plane, obtained with the $0-1$ test for chaos, for $f=0.083$ and $\Omega=0.8$.

In Figure 5 it is shown the projection of the basin of attraction in displacement vs velocity plane, for $f=0.083$ and $\Omega=0.9$. The results display a behavior very similar to the previous case, although regular responses appear embedded in previous chaotic regions.

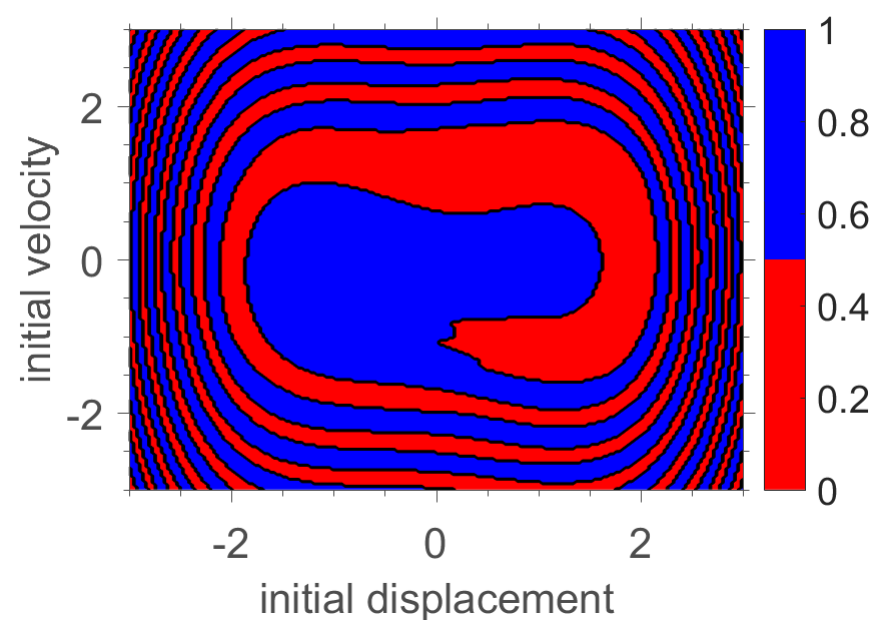

Figure 5. Projection of the basins of attraction in the displacement vs velocity plane, obtained with the $0-1$ test for chaos, for $f=0.083$ and $\Omega=0.9$. 


\section{Final remarks}

This paper addresses the nonlinear dynamic behavior of a bi-stable piezoelectric energy harvester regarding chaos incidence over system output voltage profile. Bifurcation diagrams and basins of attraction (obtained with aid of 0-1 test for chaos) are employed to distinguish the dynamic behavior of the system between regular and chaotic. The analyzes reveal a very rich dynamic behavior, with the coexistence of stable solutions and dense regions of chaos.

This work is one more in a series where the authors explore in detail the dynamics of the system of interest aiming to better know their physics to optimize the energy recovery process. In a next step, the authors intend to analyze the effect of parameter uncertainties on the system dynamic behavior.

\section{Acknowledgments}

The authors are indebted to the Brazilian agencies CNPq, CAPES, and FAPERJ for the financial support given to this research.

\section{References}

[1] F. Cottone, H. Vocca and L. Gammaitoni, Nonlinear Energy Harvesting, Physical Review Letters, 102, 080601 DOI 10.1103/PhysRevLett.102.080601 (2009)

[2] P. Spies, M. Pollak and L. Mateu, Handbook of Energy Harvesting Power Supplies and Applications Pan Stanford (2015)

[3] A. Pfenniger, A. Stahel, V. M. Koch, D. Obrist and R. Vogel, Energy harvesting through arterial wall deformation: A FEM approach to fluid-structure interactions and magneto-hydrodynamics, Applied Mathematical Modelling, 38, 3325-3338 DOI 10.1016/j.apm.2013.11.051 (2014)
[4] S. Nabavi and L. Zhang, MEMS piezoelectric energy harvester design and optimization based on Genetic Algorithm In: 2016 IEEE International Ultrasonics Symposium (IUS) DOI 10.1109/ultsym.2016.7728786 (2016)

[5] A. Cunha Jr, Enhancing the performance of a bistable energy harvesting device via cross-entropy method https://hal.archives-ouvertes.fr/hal01531845

[6] J. V. L. L. Peterson, V. G. Lopes and A. Cunha Jr, Maximization of the electrical power generated by a piezo-magneto-elastic energy harvesting device In: XXXVI Congresso Nacional de Matemática Aplicada e Computacional DOI 10.5540/03.2017.005.01.0200 (2016)

[7] V. G. Lopes, J. V. L. L. Peterson and A. Cunha Jr, Numerical study of parameters influence over the dynamics of a piezo-magneto-elastic energy harvesting device In: $X X X V I I$ Congresso Nacional de Matemática Aplicada e Computacional DOI 10.5540/03.2018.006.01.0407 (2017)

[8] V. G. Lopes, J. V. L. L. Peterson and A. Cunha Jr, On the nonlinear dynamics of a bi-stable piezoelectric energy harvesting device In: 24th $A B C M$ International Congress of Mechanical Engineering DOI 10.26678/abcm.cobem2017.cob17-1570 (2017)

[9] A. Erturk, J. Hoffmann and D. J. Inman, $A$ piezomagnetoelastic structure for broadband vibration energy harvesting, Applied Physics Letters, 94254102 DOI 10.1063/1.3159815 (2009)

[10] G. A. Gottwald and I. Melbourne, The 0-1 Test for Chaos: A review In: C. Skokos, G.A. Gottwald, and J. Laskar (Eds.). Chaos Detection and Predictability, Springer Lecture Notes in Physics 915 DOI 10.1007/978-3-662-48410-4 (2016)

[11] D. Bernardini and G. Litak, An overview of 0-1 test for chaos, Journal of the Brazilian Society of Mechanical Sciences and Engineering, 38 14331450 DOI 10.1007/s40430-015-0453-y (2016) 